\title{
Abstract Selection
}

\begin{abstract}
Autonomic reflexes and non-allergic rhinitis. Jones, A. S. Department of Otolaryngology, University of Liverpool, Royal Liverpool University Hospital, United Kingdom. Allergy (1997), Vol. 52 (36 Suppl), pp. 14-9.

Functions traditionally ascribed to the nose include warming humidification, and filtration of inspired air prior to its passage to the lower respiratory tract. The nose thus conditions inspired air, making it suitable for pulmonary gas exchange. In order to carry out these functions the nose is subject to a complex series of reflexes mediated via the autonomic nervous system. The effector tissues are the nasal blood vessels and glands. Perennial nonallergic rhinitis may be divided into two types based on the presence or absence of eosinophilia in nasal secretions Much circumstantial evidence suggests that non-eosinophilic non-allergic rhinitis (NENAR) may be a disease of autonomic imbalance. In a series of studies carried out at the University of Liverpool, patients with perennial non-allergic rhinitis and NENAR in particular, were found to have qualitative and quantitative abnormalities in their nasal response to various stimuli as manifested by changes in nasal patency. The nasal response to axillary pressure is much reduced in NENAR patients compared with normal controls, and the normal decrease in nasal resistance in response to standing is abrogated. Isometric exercise has little effect in normal subjects but those with NENAR demonstrate an increase in nasal resistance. A similar effect is seen in response to the cold pressor test. Of great importance for therapy is the effect of topical fluticasone propionate in patients with NENAR. Treatmen normalizes the damaged nasal reflexes seen in this condition, whereas placebo has no effect. However, abnormalities in other non-nasal autonomic reflexes (systemic parameters) are not affected by treatment. Author.
\end{abstract}

Isolation of a novel human homologue of the gene coding for echinoderm microtubule-associated protein (EMAP) from the Usher syndrome type la locus at 14q32. Eudy, J. D., Ma Edmonds, M. Yao, S. F., Talmadge, C. B., Kelley, P. M., Weston, M. D. Kimberling W. J., Sumegi, J. Department of Pathology and Microbiology, University of Nebraska Medical Center, Omaha 68198, USA. Genomics (1997) July 1, Vol. 43 (1), pp. 104-6.

Usher syndrome type 1 (USH1) is an autosomal recessive, genetically heterogeneous disorder causing severe congenital deafness, retinitis pigmentosa, and vestibular dysfunction. The USH1a locus located on $14 \mathrm{q} 32$ has been linked to the genetic markers D14S250 and D14S78. Using D14S250 and D14S78, we have isolated two nonchimeric YACs, $878 \mathrm{~g} 10$ and $844 \mathrm{~g} 2$, and a single BAC (135i20) and PAC (194e17) clone and have arranged them into a contig spanning over the D14S250 and D14S78 markers. The analysis of the YACs, BAC, and PAC revealed that the physical distance between D14S250 and D14S78 is less than $25 \mathrm{~kb}$. Iterative cDNA library screening initiated with the EST 219670 found in the vicinity of the D14S78 marker yielded a cDNA contig. The nucleotide sequence of the cDNA encodes a protein of 717 amino acids in length, showing a high level of homology to the Echinoderm $77 \mathrm{kDa}$ microtubule-associated protein (EMAP). The human homologue of Echinoderm microtubule-associated protein defines a novel human gene. We propose that the human EMAP is a strong candidate for the USH1a gene based on its genomic location and the proposed function of the protein. Author.

Stereotactic radiosurgical treatment of sphenopalatine neuralgia Case report. Pollock, B. E., Kondziolka, D. Department of Neurological Surgery, University of Pittsburgh Medical Center, Pennsylvania 15213, USA. Journal of Neurosurgery (1997) September, Vol. 87 (3), pp. 450-3.

Sphenopalatine neuralgia is a rare craniofacial pain syndrome that is characterized by unilateral pain in the orbit, mouth, nose, and posterior mastoid process During attacks of pain, vasomotor activity often results in ipsilateral nasal drainage, eye irritation, and lacrimination. The authors present a patient with a 15 -year history of sphenopalatine neuralgia who underwent stereotactic radiosurgery targeted at the sphenopalatine ganglion, with initial pain relief, and repeated radiosurgery 17 months later for partial pain recurrence. Two years following radiosurgery, the patient is pain free, no longer suffering from nasal discharge and eye irritation. Author.

Severe cervical dysplasia and nasal cartilage calcification following prenatal warfarin exposure. Howe, A. M., Lipson, A. H., de Silva, M., Ouvrier, R., Webster, W. S. Department of Anatomy and Histology, University of Sidney, NSW, Australia. American Journal of Medical Genetics (1997) September 5, Vol. 71 (4), pp. 391-6. We present an infant who was exposed to warfarin throughout pregnancy and has warfarin embryopathy. When the child was examined radiologically at 20 months areas of calcification were visible in the septal and alar cartilages of the small external part of the nose. The location of this ectopic calcification is consistent with that seen in an animal model of the warfarin embryopathy. It supports the hypothesis that warfarin interferes with the prenatal growth of the cartilaginous nasal septum by inhibiting the normal formation of a vitamin $\mathrm{K}$-dependent protein that prevents calcification of cartilage. The child also had severe abnormalities of the cervical vertebrae and secondary damage to the spinal cord. Cervical vertebral anomalies are a relatively common finding in the warfarin embryopathy and in the related Binder syndrome. Author.

New gene for autosomal recessive non-syndromic hearing loss maps to either chromosome 3q or 19p. Chen, A., Wayne, S., Bell, A., Ramesh, A., Srisailapathy, C. R. Scott, D. A., Sheffield, V. C., Van Hauwe, P., Zbar, R. I., Ashley, J., Lovett, M., van Camp, G., Smith, R. J. Department of Otolaryngology, University of Iowa, Iowa City 52242, USA. American Journal of Medical Genetics (1997) September 5, Vol. 71 (4), pp. 467-71

Autosomal recessive non-syndromic hearing loss (ARNSHL) is the most common form of prelingual inherited hearing impairment. A small consanguineous family with this disorder was ascertained through the Institute of Basic Medical Sciences in Madras, India. Conditions such as rubella, prematurity, drug use during pregnancy, perinatal trauma, and meningitis were eliminated by history. Audiometry was performed to confirm severe-toprofound hearing impairment in affected persons. After excluding linkage to known DFNB genes, two genomic DNA pools, one from the affected persons and the other from their non-affected siblings and the parents, were used to screen 165 polymorphic markers evenly spaced across the autosomal human genome. Two regions showing homozygosity-by-descent in the affected siblings were identified on chromosomes 3q21.3-q25.2 and 19p13.3-p13.1, identifying one (or possibly both) as the site of a novel ARNSHL gene. Author

Pain experienced by patients with terminal head and neck carcinoma. Talmi, Y. P., Waller, A., Bercovici, M., Horowitz, Z., Pfeffer, M. R., Adunski, A. Kronenberg, J. Department of Otolaryngology-Head and Neck Surgery, The Chaim Sheba Medical Center, Tel Hashomer, Israel. Cancer (1997) September 15, Vol. 80 (6), pp. 1117-23.

BACKGROUND: Pain is one of the most feared consequences of cancer and is experienced by up to 80 per cent of patients with head and neck carcinoma (HNC). Pain in terminal HNC patients is common and often defined as severe. This study evaluated the effectiveness of the World Health Organization (WHO) analgesic ladder in the treatment of a cohort of terminal HNC patients METHODS: The authors prospectively evaluated 62 consecutive 
terminal HNC patients admitted to the Chaim Sheba Medical Center Tel Hashomer Hospice or the general hospital. Data pertaining to tumour origin, spread, treatment, and results were defined. Pain was assessed with the McGill Pain Questionnaire, using a 10-point visual analogue scale (VAS) and a body map. Pain was diagnosed according to cause and type. Treatment was selected according to the guidelines provided in the WHO analgesic ladder. RESULTS: Only 10 patients suffered from pain that was not locoregional. The results of the VAS score were available in the first reading in all patients with pain $(n=48)$, with a mean of 4.7 (standard deviation (SD) \pm 2.0 ). A mean second VAS score obtained 72 hours after the first was $1.9(\mathrm{SD} \pm 1.1)$. The difference between the two scores was statistically significant $(p<0.001)$. A third score was available for only six patients, with a mean of 1.6. Only two patients did not experience improvement of pain after 72 hours of treatment; both of these patients had bony involvement with tumour. Thirty-one patients ( 65 per cent) were diagnosed with pain of nociceptive origin; these patients were categorized as having actual nociceptive pain (22), nociceptive nerve pain (six), or referred pain to the ear (three). Nonnociceptive pain of neuropathic origin was noted for only six patient $(12.5$ per cent). Pain that could not be well defined but was responsive to opioid analgesic treatment was noted for 11 patients $\mathrm{A}$ different form of non-cancer-related pain was noted for only one patient. CONCLUSIONS: Patients were treated for pain according to the WHO analgesic ladder. They received adequate narcotic analgesics and supportive measures that allowed significant reduction of pain in nearly all cases, with acceptable side effects Author.

Pathophysiology and therapy for allergic and nonallergic rhinitis: an updated review. Levenson, T., Greenberger, P. A. Ernest S. Bazley Asthma and Allergic Diseases Center. Department of Medicine, Northwestern Memorial Hospital, Chicago, Illinois, USA. Allergy and Asthma Procedures (1997) July-August, Vol. 18 (4), pp. 213-20.

Symptoms from rhinitis can be assessed to be more troubling to the patient than symptoms from asthma. Further, the array of bioactive mediators, cytokines, and cells in nasal mucosa suggests that rhinitis is a much more complex condition than formerly thought. The presence of allergic rhinitis is a risk factor for emergence of asthma. Topical nasal corticosteroids have a relatively flat dose-response curve and have onset of action within one to two days for some patients. Allergen immunotherapy remains the only immunomodulator for patients with allergic rhinitis with the possible exception of long-term administration of nasal corticosteroids. Author.

The P1 component of the middle latency auditory potential may differentiate a brainstem subgroup of Alzheimer disease. Green, $J$. B., Burba, A., Freed, D. M., Elder, W. W., Xu, W. Edward Hines, Jr. Veterans Hospital, Hines, Illinois 60141, USA Alzheimer Diseases and Associated Disorders (1997) September, Vol. 11 (3), pp. 153-7.

The P1 component of the middle latency auditory evoked potential (MLAEP) was found to be absent in 47.5 per cent of 101 patients with Alzheimer disease (AD). Lack of a P1 component recently has been associated with a more rapid decline in cognitive performance. The blink reflex (BR) was tested in 36 patients with probable $\mathrm{AD}$ and 17 elderly control subjects All subjects also underwent $\mathbf{P 1}$ recording. Patients lacking a P1 potential showed a significant increase in latency of the contralateral R2 response of the BR. The P1 component and the $R 2$ response may differentiate a subgroup of $A D$ patients with involvement of the brainstem, especially the reticular formation. Author.

Familial paragangliomas: linkage to chromosome $11 \mathrm{q} 23$ and clinical implications. Milunsky, J., DeStefano, A. L., Huang, X. L., Baldwin, C. T., Michels, V. V., Jako, G., Milunsky, A. Center for Human Genetics and Department of Pediatrics Boston University School of Medicine, Massachusetts 02118, USA. American Journal of Medical Genetics (1997) October 3, Vol. 72 (1), pp. 66-70. Familial paragangliomas (PGL), or glomus tumours are slowgrowing, highly vascular, generally benign neoplasms usually of the head and neck that arise from neural crest cells. This rare autosomal-dominant disorder is highly penetrant and influenced by genomic imprinting through paternal transmission. Timely detection of these tumours affords the affected individual the opportunity to avoid the potential morbidity associated with surgical removal, and mortality that may accompany local and distant metastases. Linkage to two distinct chromosomal loci, $11 \mathrm{q} 13.1$ and $11 \mathrm{q} 22.3-\mathrm{q} 23$, has been reported, suggesting heterogeneity. We evaluated three multigenerational families with hereditary PGL, including 19 affected, and 59 unaffected and potentially at-risk individuals. Numerous microsatellite markers corresponding to each candidate region were tested in all members of the three families Confirmation of linkage to 11q23 was established in all three families. The inheritance pattern was consistent with genetic imprinting. Using these data, we were able to provide presymptomatic diagnosis with subsequent removal of tumour from one individual, and to start several others on an MRI surveillance protocol. Author.

Lipoprotein-stimulated surfactant secretion in alveolar type II cells: mediation by heterotrimeric G proteins. Pian, M. S., Dobbs, L. G. Department of Pediatrics University of California, San Francisco 94143-0130, USA. American Journal of Physiology (1997) September, Vol. 273 (3 Pt 1), pp. L634-9.

Low- and high-density lipoproteins (LDL and HDL, respectively) stimulate alveolar type II cells to secrete surfactant. Increases in phosphoinositide hydrolysis, cytosolic $\mathrm{Ca}^{+}$, and membraneassociated protein kinase $\mathrm{C}$ activity precede LDL- and HDLstimulated secretion. We report three lines of evidence supporting the hypothesis that Gi mediates LDL- and HDL-stimulated surfactant secretion and signal transduction in type II cells. First, pertussis toxin (PTX) inhibited secretion stimulated by the apolipoprotein ligands for either the LDL receptor or the HDL binding protein. Second, PTX inhibited protein kinase $\mathrm{C}$ activity in cell membranes stimulated by LDL or HDL. Third, treatment of cell membranes with LDL or HDL inhibited PTX-catalyzed labelling of substrates corresponding in molecular mass to $\mathrm{Gi}$ alpha. These observations suggest that receptor-mediated activation of Gi is required for LDL- and HDL-stimulated secretion and that LDL and HDL activate Gi. These studies in type II cells are the first to support the hypothesis that Gi mediates the effects of LDL or HDL on important phenotype-specific functions of differentiated cells. Author.

Investigation into the increase in hay fever and eczema at age 16 observed between the 1958 and 1970 British birth cohorts. Butland, B. K., Strachan, D. P., Lewis, S., Bynner, J., Butler, N., Britton, J. Department of Public Health Sciences, St George's Hospital Medical School, London. British Medical Journal (1997) September 20, Vol. 315 (7110), pp. 717-21.

OBJECTIVE: To investigate whether changes in certain perinatal and social factors explain the increased prevalence of hay fever and eczema among British adolescents between 1974 and 1986. DESIGN: Two prospective birth cohort studies SETTING: England, Wales and Scotland. SUBJECTS: 11,195 children born 3-9 March 1958 and 9,387 born 5-11 April 1970. MAIN OUTCOME MEASURES: Parental reports of eczematous rashes and of hay fever or allergic rhinitis in the previous 12 months at age 16. RESULTS: The prevalence of the conditions over the 12 month period increased between 1974 and 1986 from 3.1 per cent to 6.4 per cent (prevalence ratio 2.04 ( 95 per cent confidence interval 1.79 to 2.32)) for eczema and from 12.0 per cent to 23.3 per cent (prevalence ratio) 1.93 (1.82 to 2.06)) for hay fever. Both conditions were more commonly reported among children of higher birth order and those who were breastfed for longer than one month. Eczema was more commonly reported among girls and hay fever among boys. The prevalence of hay fever decreased sharply between social classes I and $\mathrm{V}$, increased with maternal age up to the early $30 \mathrm{~s}$, and was lower in children whose mothers smoked during pregnancy. Neither condition varied significantly with birth weight. When adjusted for these factors the relative odds of hay fever (1986 v 1974) increased from 2.23 (2.05 to 2.43) to 2.40 (2.19 to 2.63 ). Similarly, the relative odds of eczema rose from 2.02 (1.73 to 2.36 ) to 2.14 (1.81 to 2.52 ). CONCLUSIONS: Taken together, changes between cohorts in sex, birth weight, birth order, maternal age, breast feeding, maternal smoking during 
pregnancy, and father's social class at birth did not seem to explain any of the observed rise in the prevalence of hay fever and eczema. However, correlates of these factors which have changed over time may still underlie recent increases in allergic disease. Author.

The optimal number of pleural biopsy specimens for a diagnosis of tuberculous pleurisy. Kirsch, C. M., Kroe, D. M., Azzi, R. L., Jensen, W. A., Kagawa, F. T., Wehner, J. H. Division of Respiratory and Critical Care Medicine, Santa Clara Valley Medical Center, San Jose, California, USA. Chest (1997) September, Vol. 112 (3), pp. 702-6.

STUDY OBJECTIVES: To determine the optimal number of pleural biopsy (PLBX) specimens for a diagnosis of tuberculous pleurisy. DESIGN: Retrospective review. SETTING: County hospital. METHODS: We reviewed all percutaneous needle biopsy specimens of the parietal pleura in 30 patients who had tuberculous pleurisy. Data are reported as mean $\pm S E M$ and statistical comparisons are done with the Mann-Whitney test. We accepted $p<0.005$ as statistically significant. RESULTS: The number of biopsy specimens obtained from each patient ranged from four to 10 with one sample submitted for mycobacterial culture and the rest submitted for histologic study. Sixty per cent of patients had pleural cultures positive for Mycobacterium tuberculosis and 80 per cent had diagnostic histology. Overall pleural biopsy sensitivity (histology and culture) for tuberculous pleurisy was 87 per cent. On average, 40.4 per cent \pm 4.7 per cent of all PLBX specimens contained pleura. Diagnostic PLBX procedures compared to false-negative procedures produced more tissue specimens $(7.1 \pm 0.3$ vs $4.8 \pm 0.5, p=0.005)$ containing more pleural specimens $(2.4 \pm 0.2$ vs $0.8 \pm 0.5, p=0.01)$. If only PLBX procedures yielding more than six tissue specimens $(n=18)$ or more than two pleural specimens $(n=12)$ were analyzed, then the diagnostic sensitivity of PLBX for pleural tuberculosis was 100 per cent. There seemed to be a direct relationship between the sensitivity of PLBX and the number of specimens submitted. CONCLUSIONS: The sensitivity of percutaneous needle biopsy for diagnosis of tuberculous pleurisy is highest when more than six specimens are obtained which, on average, contain more than two specimens of parietal pleura. There are no conclusive data indicating how many tissue specimens to submit for mycobacterial culture, but one specimen seems sufficient. Author.

Characterization of leprosy based on the nasal lipid profile Srikanth, N. C., Gangadhar, S., Reddy, Y. N., Apte, S. S. Lakshmipathy, V., Apparao, A. V., Krishna, D. R., Prabhakar M. C. Hansen's Laboratory, University College of Pharmaceutical Sciences, Kakatiya University, Warangal. Indian Journal of Leprosy (1997) April-June, Vol. 69 (2), pp. 179-81.

While extracting the $M$. leprae from the nasal flushings of leprosy patients it was found that these organisms were trapped in the waxy layer, between the aqueous and the chloroform layers. Thin layer chromotography (TLC) analysis of this layer, using chloro- form-methanol-water system, revealed different spots when sprayed with acid alcohol and heated at 160 degrees $C$. The TLC profile of lipids of lepromatous and borderline (MB according to the WHO terminology) leprosy patients was distinctly different from that of tuberculoid leprosy patients and normal human volunteers. A simple, economical and fast procedure to characterize patients belonging to different spectra has been developed. Author.

Newborn hearing screening by otoacoustic emissions and automated auditory brainstem response. Doyle, K. J., Burggraaff, B., Fujikawa, S., Kim, J. Department of Otolaryngology-Head and Neck Surgery, University of California Irvine, Orange 92668 , USA. International Journal of Pediatric Otorhinolaryngology (1997) August 20, Vol. 41 (2), pp. 111-9.

The aim of this study is to compare pass rates for two different hearing screening methods in well newborns as a function of age. Hearing screening tests were performed on 400 ears in 200 healthy newborn infants at the University of California-Irvine Medical Center. The screening methods used were automated auditory brainstem response (ABR) and click evoked otoacoustic emissions (EOAE). The infants' ages ranged from five to $120 \mathrm{~h}$, with an average age of $24 \mathrm{~h}$. Overall, 88.5 per cent of ears passed the ABR screen, and 79 per cent passed the EOAE screen. There was no significant difference in the ABR pass rate for infants aged 0-24 $\mathrm{h}$ of age as compared with infants aged $>24 \mathrm{~h}$ compared with the group aged $0-24 \mathrm{~h}(p<0.01)$. Results are compared with earlier studies and implications for universal hearing screening are discussed. Author.

The relation between age at the time of cochlear implantation and long-term speech perception abilities in congenitally deaf subjects. Snik, A. F., Makhdoum, M. J., Vermeulen, A. M., Brokx, J. P., van den Broek, P. Department of Otorhinolaryngology, University Hospital, Nijmegen, The Netherlands. International Journal of Pediatric Otorhinolaryngology (1997) August 20, Vol. 41 (2), pp. 121-31.

The issue of whether an upper age limit should be set for cochlear implantation in congenitally deaf subjects has often been debated. To gain more insight, the speech perception abilities were analyzed of 12 congenitally deaf subjects whose age at the time of cochlear implantation ranged from four to 33 years. Subjects implanted during adulthood only showed progress during the first few months after the speech processor had been fitted and their long-term results were poor compared to those of children implanted early in life. This latter group showed steady improvement over the whole evaluation period. The present results support the notion that the earlier in life implantation is performed, the better the development of speech perception. Based on the progress-over-time profiles and data on actual daily use of the cochlear implant, it can be suggested that implantation of congenitally deaf subjects during or after puberty offers only limited benefit. Author. 